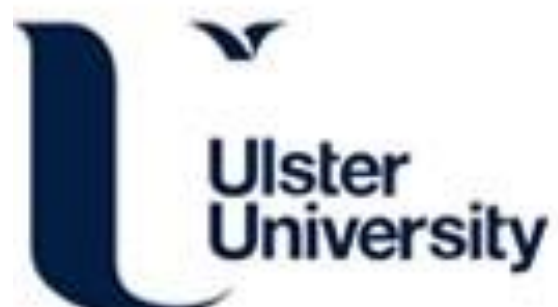

Analysis of a Class of Low-Dimensional Models of Mutation and Predation

Abernethy, G., \& McCartney, M. (2016). Analysis of a Class of Low-Dimensional Models of Mutation and Predation. International Journal of Bifurcation and Chaos, 26(11), 1630029-1-13.

https://doi.org/10.1142/S0218127416300299

Link to publication record in Ulster University Research Portal

\section{Published in:}

International Journal of Bifurcation and Chaos

Publication Status:

Published (in print/issue): 31/10/2016

DOI:

10.1142/S0218127416300299

\section{Document Version}

Author Accepted version

\section{General rights}

Copyright for the publications made accessible via Ulster University's Research Portal is retained by the author(s) and / or other copyright owners and it is a condition of accessing these publications that users recognise and abide by the legal requirements associated with these rights.

\section{Take down policy}

The Research Portal is Ulster University's institutional repository that provides access to Ulster's research outputs. Every effort has been made to ensure that content in the Research Portal does not infringe any person's rights, or applicable UK laws. If you discover content in the Research Portal that you believe breaches copyright or violates any law, please contact pure-support@ulster.ac.uk. 


\title{
Analysis of a Class of Low-Dimensional Models of Mutation and Predation
}

\author{
Gavin M Abernethy*and Mark McCartney $\mathrm{PhD}^{\dagger}$ \\ School of Computing and Mathematics, \\ Ulster University, \\ Jordanstown Campus, Shore Road, Newtownabbey, \\ Co. Antrim, BT37 0QB, \\ UK
}

September 2, 2016

\begin{abstract}
We consider a class of simple two-dimensional discrete models representative of a system incorporating both mutation and predation. A selection of analytic and numerical results are presented, classifying the dynamic behaviour of the system by means of Lyapunov exponents over a biologically-reasonable region of parameter space, and illustrating the occurrence of hyperchaos and a Neimark-Sacker bifurcation producing regions of quasiperiodicity.
\end{abstract}

\section{Introduction}

Mathematical modelling of systems of species in predator-prey relationships begins with Volterra's pair of ordinary differential equations (independently formulated by the chemist Lotka the previous year) when contemplating the variation in fish stocks in the Mediterranean Sea in 1926 [Vol26]. In their simplest form, the populations of a prey $x$ and predator $y$ are governed by:

$$
\begin{aligned}
& \frac{\mathrm{d} x}{\mathrm{~d} t}=x(1-y) \\
& \frac{\mathrm{d} y}{\mathrm{~d} t}=a y(x-1) .
\end{aligned}
$$

This marks the addition of predator-prey modelling to the mathematical science of population dynamics begun by Malthus in the late $18^{\text {th }}$ century. Since then, more sophisticated versions such as those with Holling [Hol59], Beddington [Bed75] and Ratio-Dependent [AG89] predator functional responses have been developed and many investigations have considered variations of these models. For example [AEEME09, JY06, HL11, BCPS01, HIT10, HTZ11] have studied two-dimensional discrete models, with bifurcation analysis from a dynamical systems perspective proving a popular approach in recent years.

In this paper we consider a class of models derived from the following discrete predator-prey model presented in [Smi68]:

$$
\begin{aligned}
x_{n+1} & =a x_{n}-\frac{(a-1)}{b} x_{n}^{2}-c x_{n} y_{n} \\
y_{n+1} & =\frac{a}{b} x_{n} y_{n} .
\end{aligned}
$$

The above system is also used as an example for bifurcation analysis in Kuznetsov's textbook [Kuz13]. Our class of models takes this system as a basis and superimposes upon it a very simple representation of mutation by constant diffusion from the prey species to the predator. Some authors have looked at coupling two logistic maps to model spatial structure [Llo95, Fro92], however these cases model a single species that can freely move in a two-patch environment. In this paper we instead consider mutation in one direction only, which could be used to represent a fraction of the population at each time step mutating to a competing predatory subspecies, or simply developing cannibalistic tendencies that they will pass to their offspring. This provides an alternative to most cannibalistic studies which typically employ an age-stratification

\footnotetext{
*abernethy-g1@email.ulster.ac.uk
}

†m.mccartney@ulster.ac.uk 
approach [BLR10, KT03, SJL10]. Some versions of our model also include interspecific competition between $x$ and $y$, and logistic growth for the predator species with an increase to the reproductive parameter facilitated by the presence of prey. The general form is described in the next section.

Recently, Ghaziani [KG14] provided a comprehensive analysis of a similar nature for another system in this tradition. This model differs from ours in a number of regards, namely it featured a Lotka-Volterra functional response, no mutation or interspecific competition, and was derived from a continuous-time model via the Euler scheme with a constant natural death rate for the predator.

\section{Method}

\subsection{The Models}

We consider simple models of two interacting species with the intention of exploring the simplest possible model that encapsulates both predation and mutation. Discrete-time systems are typically easier to manipulate than their continuous-time counterparts. Therefore, we use discrete-time logistic growth as the basis for our model. We look at three versions of a model where only one species, $x$, is initially populated. This is the evolutionary "parent" species, and it produces a "child" $y$ via mutation. The trophic interaction is that this child shall be the designated predator, capable of increasing its reproduction by hunting the prey species $x$. To maintain simplicity, we shall assume a constant rate of mutation $p$. Furthermore, we make the assumption that both species have a lifetime of a single timestep, with mutation occuring at the moment of reproduction, and predation taking place upon the juveniles of $x$.

Our model, therefore, follows the general form:

$$
\begin{aligned}
& x_{n+1}=(1-p) F_{1}\left(x_{n}, y_{n}\right) x_{n}-g\left(x_{n}, y_{n}\right) y_{n} \\
& y_{n+1}=p F_{1}\left(x_{n}, y_{n}\right) x_{n}+F_{2}\left(g, x_{n}, y_{n}\right) y_{n}
\end{aligned}
$$

where $F_{1}, F_{2}$ are the density-dependent growth functions of $x$ and $y$ respectively, $g$ is the predator functional response, and the domain is $[0,1) \times[0,1)$. As noted above, we shall use logistic growth for the prey species, and the control parameter will be denoted by $r$.

The next modelling choice is the predator functional response, which determines the negative effect of predation upon the prey species. The best form for the functional response has been the subject of substantial debate in the ecological literature [AG00], but following (3) in all versions of our model we shall content ourselves with the simplest reasonable form, which is linear in $x$, and termed Lotka-Volterra dynamics in reference to the corresponding continuous-time model. Thus, $g\left(x_{n}, y_{n}\right)=-c x_{n}$, where parameter $c$ controls the rate of predation.

The variants of the model are differentiated by the following features:

Model 1, the simplest, incorporates competition between the two species, and the predator is fully dependent on the prey to survive, with its growth function equivalent to its numerical response to $x$ and directly proportional to the functional response. One might say that it is a "pure predator", as it is unable to obtain resources in any other manner. This is altered in Models 2 and 3, where $y$ can survive independently of its prey by means of logistic growth, but the presence of $x$ is of benefit to $y$ and allows it to reproduce more effectively. (A pair of logistic maps coupled by a similar mechanism were studied in [ES08a].) In order that the growth of both species remain comparable, we scale the reproductive parameter of $y$ by $1 / 2$ compared to that of $x$ in Model 3. In Model 2, if this were to remain the case it would result in $y$ being competitively unviable, so we scale its growth by $2 / 3$ instead. This is because Model 2 includes a simple representation of interspecific competition, whereas in Model 3 this is discarded and we consider instead the situation where the predator species has mutated sufficiently from its prey-parent such that they now occupy two separate ecological niches.

\section{Model 1.}

$$
\begin{aligned}
x_{n+1} & =\max \left((1-p) r x_{n}\left(1-x_{n}-y_{n}\right)-c x_{n} y_{n}, 0\right) \\
y_{n+1} & =\max \left(p r x_{n}\left(1-x_{n}-y_{n}\right)+r x_{n} y_{n}, 0\right)
\end{aligned}
$$

\section{Model 2.}

$$
\begin{aligned}
& x_{n+1}=\max \left((1-p) r x_{n}\left(1-x_{n}-y_{n}\right)-c x_{n} y_{n}, 0\right) \\
& y_{n+1}=\max \left(p r x_{n}\left(1-x_{n}-y_{n}\right)+\frac{2 r}{3}\left(1+x_{n}\right) y_{n}\left(1-x_{n}-y_{n}\right), 0\right)
\end{aligned}
$$




\section{Model 3.}

$$
\begin{aligned}
& x_{n+1}=\max \left((1-p) r x_{n}\left(1-x_{n}\right)-c x_{n} y_{n}, 0\right) \\
& y_{n+1}=\max \left(p r x_{n}\left(1-x_{n}\right)+\frac{r}{2}\left(1+x_{n}\right) y_{n}\left(1-y_{n}\right), 0\right)
\end{aligned}
$$

Note that for each equation we implement a maximum function in order to prevent the occurrence of negative populations.

The remaining structure of this work is as follows: In Section 3, we shall present the results of a numerical investigation of these models with a defined, constant mutation rate of $p=10^{-3}$. The emphasis shall be on the global behaviour of the system across the $(c, r)$ parameter space from the viewpoint of ecological modelling and population dynamics. Despite their relatively simple description, such nonlinear models are often highly resistant to yielding general analytic results. Therefore we will frequently compare the computational results of our simulations to analytic statements concerning the limiting cases of the models as $p \rightarrow 0$, in order to obtain insight into the mathematics underlying our observations. The limiting case of Model 3 retains particularly interesting dynamics, which shall be explored in more detail in Section 4 . Section 5 presents the conclusions.

\subsection{Numerical Methods}

For each version of the model we present images of the qualitative behaviour of the system in the parameter space $(c, r) \in$ $[0,10) \times[0,5)$. These are created by numerical calculation of the maximal (characteristic) Lyapunov exponent, denoted by $\lambda$ throughout, using the algorithm and error estimates for two-dimensional systems provided in Sprott's textbook [Spr03]. For each set of parameters, the algorithm is performed with $10^{5}$ transient iterations and then a further $10^{6}$ calculations are used to calculate the maximal exponent. Therefore, taking the error approximation $1 / \sqrt{N}$, we will not assume chaos unless $\lambda>10^{-3}$. The resulting behaviour is then classified in the following manner:

i). $\lambda>10^{-3}$ indicates chaotic behaviour.

ii). When $|\lambda|<10^{-3}$, we determine the dynamics to be quasiperiodic, where the maximal Lyapunov exponent is identically zero for extended regions. Note that this is also the case at infinitesimal points of bifurcation, which is sometimes visible due to the coarse range of classification.

iii). When $\lambda<-10^{-3}$, ordered behaviour occurs and we perform a test over the final $10^{4}$ iterations to determine if the system has reached a fixed point within a $10^{-6}$ tolerance in each dimension. Thus, for $\lambda<-10^{-3}$, we separately classify period-1 (fixed point) behaviour and periodic behaviour. Finally, all of the above scenarios are classified according to whether $x, y$ or both species are persisting.

It should be noted that, for the purposes of computation, if either population falls below a threshold of $10^{-6}$ then it is set to zero. In ecological terms this effectively sets a maximum carrying capacity of $10^{6}$ for each species, but it also ensures correct classification in the event that populations are slowly converging to zero. All numerical results are obtained using Fortran 90 with double precision arithmetic.

\section{The Case of Mutation}

\subsection{Numerical Results with $p=10^{-3}$}

For our numerical analysis, we choose a mutation rate of $p=10^{-3}$. To model a system arising from a single species, in all scenarios we start with the initial condition $x_{0}=0.1, y_{0}=0$.

In addition, we have tested the behaviour of the systems for $p=0.1, p=0.25$, and starting values of $x_{0}=0.3$. It was found that changing the initial conditions has relatively little effect except for altering the broken boundary between fixed point coexistence and the $\left(0, y^{*}\right)$ fixed point in Model 3 .

Increasing the value of $p$ tends to compress the region of co-survival in all three variants: as $p$ increases, for all $c$ the minimum value of $r$ required to allow coexistence increases, and given a sufficiently large value of $c$ the maximum $r$ for that value of $c$ which permits coexistence is reduced. For Model 2 and 3, the trend is toward flattening the border of coexistence against the axes, with the region of survival being simultaneously stretched in the $r$-axis for sufficiently low values of $c$. However for Model 1, all survival remains bounded at a maximum of $r=4$ for $c=0$, and for all $c>0$ the region of coexistence is compressed. At larger values of $p$, Model 1 also incorporates regions of quasiperiodic behaviour which, as seen in Figure 1(a), is not present when $p=10^{-3}$. The structure of the parameter space in such a case is very similar to that observed in our recent pedagogical study of a version of Model 1 with the simplified functional response $g\left(x_{n}, y_{n}\right)=-c$ [AM16]. The outcome of these experiments are not illustrated, and we shall focus on the results of the system with $p=10^{-3}$ as representative of the case of mutation. These are shown in Figure 1. 


\subsection{Model 1 Discussion}

The qualitative behaviour of Model 1 (Figure 1(a)) over the range considered is effectively independent of the consumption rate $c$, and follows the general structure expected from the 1-dimensional logistic map with control parameter $r$, passing through extinction, a stable fixed point, and then bifurcations through periodic orbits to chaos. Note that the thin green line exactly at $r=3$ is due to the Lyapunov exponent touching 0 at the first period-doubling bifurcation point, and does not indicate quasiperiodicity. Analytically, it is straightforward to confirm in the limiting case as $p \rightarrow 0$ that the existence and stability of the interior fixed point of this model is indeed unaffected by variation of $c$. However, one would expect that this parameter must have some consequence given that it cannot be removed from the system by non-dimensionalisation and, indeed, it is easily shown that the effect of increasing $c$ is to lower the size of the population of $y$ at the fixed point. In particular, when $p=0$ the coexistence fixed point takes the form $\left(\frac{1}{r}, \frac{r-2}{r+c}\right)$ and is linearly stable for all of $2<r<3$.

We note that in the case $p=10^{-3}$, the range $1<r<2$ also results in an interior period-1 orbit. This is because while the axial point $\left(1-\frac{1}{r}, 0\right)$ is the unique attractor in this region when $p=0$, for positive mutation this is forced to become a coexistence fixed point as the existence of $x$ necessitates the appearance of $y$ at the next iteration.

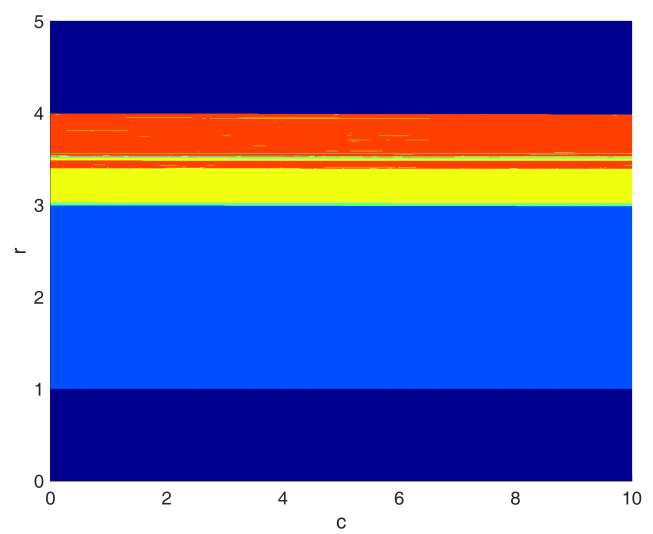

(a) Model 1

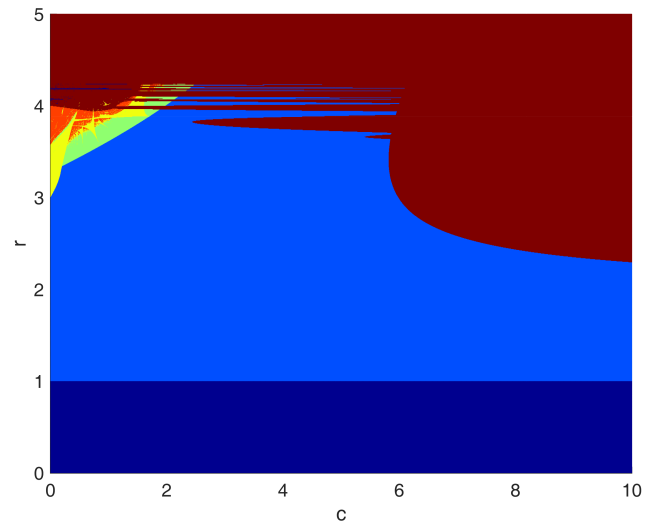

(c) Model 3

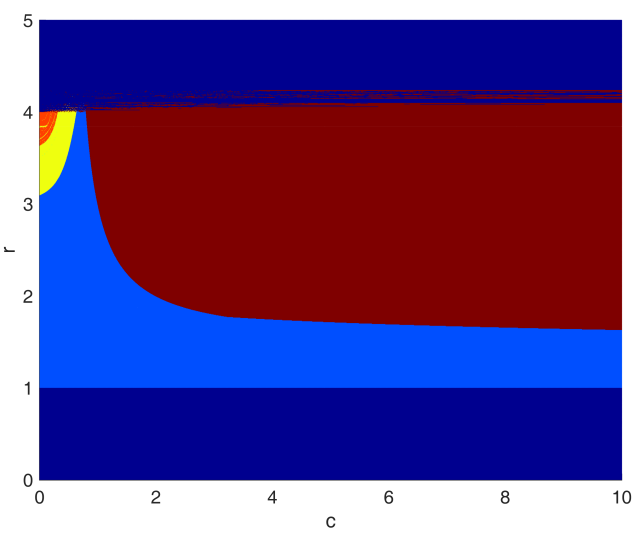

(b) Model 2

Figure 1: Qualitative Behaviour: Purple = Extinction, Blue = Coexistence Period-1, Red = y-only Period 1 [(b) and $(\mathrm{c})$ only], Green = 2D Quasiperiodicity [(c) only], Yellow $=2 \mathrm{D}$ Periodicity, Orange $=2 \mathrm{D}$ Chaos.

$p=10^{-3}$ throughout. For every set of parameter values $x_{0}=0.1, y_{0}=0.0$, and $10^{5}$ transients are discarded before the Lyapunov exponent is calculated over $10^{6}$ iterations.

\subsection{Model 2 Discussion}

For Model 2 (Figure 1(b)), the modification of the predator-child to be able to survive independently introduces a region such that if $c$ and $r$ are large then species $x$ will be driven to extinction and species $y$ will attain a fixed point by itself. In the limiting case, as $p \rightarrow 0, r=\frac{3 c}{2 c-1}$ marks the boundary of the region where the $x$-value of the coexistence fixed point is negative (and thus zero). This coincides with the axial fixed point $\left(0,1-\frac{3}{2 r}\right)$ first attaining linear stability. For $r>4$, even though $y$ has the ability to exist independently (and could survive alone up to to $r=6$ ), the region of extinction remains 
due to the possibility of $x_{n}+y_{n}>1$ for some finite $n$, which through the interspecific competition in this model will then result in the death of both species.

If we remove the possibility of $y$ 's existence, the dynamics of species $x$ reduces to the standard logistic map, which collapses (by attaining negative values) for $r>4$ and is intermittently chaotic within $a_{\infty}<r<4$, where $a_{\infty} \simeq 3.57$ denotes the accumulation point. One would expect the large variation in population numbers associated with this chaos to be biologically undesirable, especially as in hostile environments this could result in the population temporarily visiting a region of the phase space that leads to total extinction. However, in Figure 1(b) we can see that for $0.63<c<0.79$ the blue region of period-1 coexistence now extends all the way to $r=4$. For example, at $c=0.64, r=4$, species $x$ is being held at equilibrium with $x^{*}=0.105$ when in the absence of $y$ its orbit at these parameters would eventually visit an arbitrarily low value and thus result in extinction given any minimum population size. Thus, for a value of $c$ within this range it would be a beneficial form of self-regulation for the species to periodically produce a hostile mutant that would stabilise the population through predation that, in this model, could be considered a form of cannibalism. Such behaviour has been observed in other studies that considered more sophisticated models of age-based cannibalism within two-species systems [KE95, SJL10, BLR10, Hen97].

\subsection{Model 3 Discussion}

For Model 3 (Figure 1(c)), competition between the species has been removed and reproductive control parameter of $y$ has been reduced to $r / 2$. This has mostly removed the collapse of the system for $r>4$, as $x_{n}+y_{n}>1$ is no longer sufficient to elimimate both species. The region of coexistence has been extended to larger values of $c$ and $r$ from Model 2. Once again, this boundary is due to the value of $x$ becoming negative as the curve is crossed. The remaining upper bounds of the coexistence state are divided into three distinct forms:

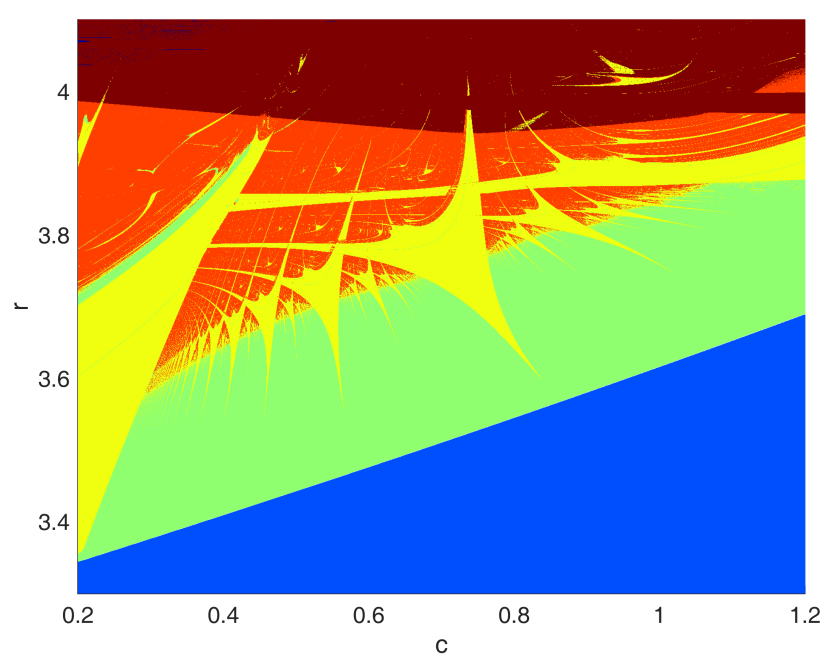

Figure 2: Detail of Figure 1(c), key as for Figure 1.

(i) The fixed point may lose stability via a flip (period-doubling bifurcation) for very small $c$ as $r$ increases.

(ii) In the range approximately $0.1<c<2$, the system undergoes Neimark-Sacker bifurcation to a closed invariant curve around the fixed point. This results in quasiperiodic behaviour which is only observed in Model 3, and Figure 2 shows the region where this occurs in greater resolution. Here we can see the Arnol'd Tongues of periodic orbits produced by phase locking at the bifurcation point. This is discussed further for the limiting case in Section 4.4.

(iii) Finally, the system moves to an axial fixed point $\left(0, y^{*}\right)$ in the range $2<c<6$ as $r$ increases past approximately 3.8. The analysis of the limiting case indicates that the interior fixed point continues to exist without further bound, and remains stable in this region and therefore this broken boundary is the result of the ecological conditions attached to the algorithms producing these images, such that $x$ is permanently set to 0 in the event that it is mapped to a negative number for even a single iteration. Together with the fractured nature of this boundary, we infer that this boundary is likely to depend on the initial conditions of the map, and this is confirmed to be the only qualitative change upon inspecting the case of $x_{0}=0.3$. 


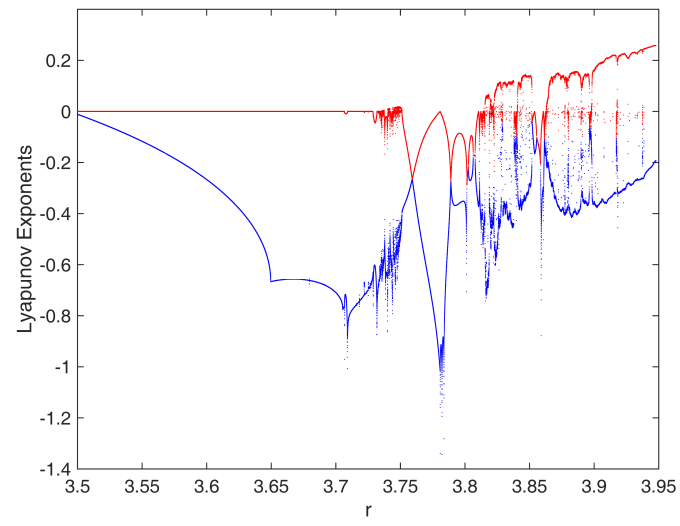

(a) Lyapunov Exponents: red denotes the Maximal Exponent. Each exponent is calculated over $10^{6}$ iterations following $10^{6}$ transients.

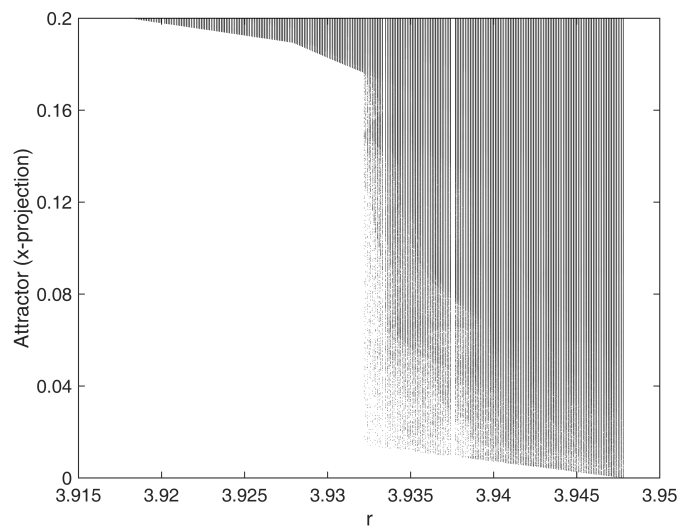

(c) Zoom on bottom-right of Feigenbaum Diagram (b), with a much larger number $\left(2 \times 10^{6}\right)$ of post-transient iterations.

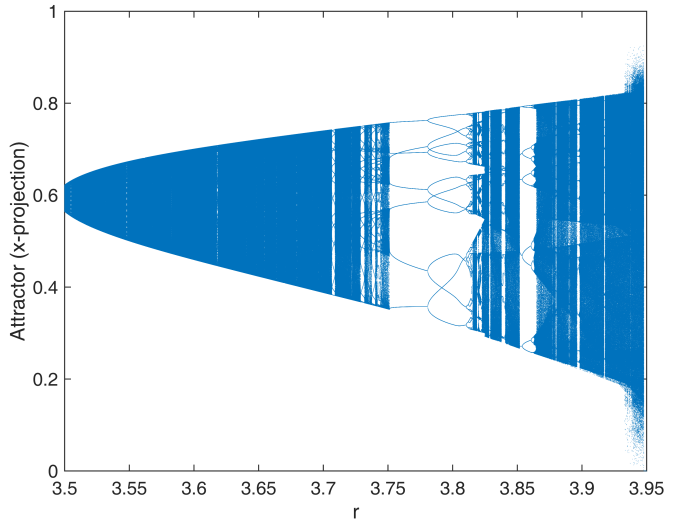

(b) Feigenbaum Diagram. Up to 1000 iterations are displayed per $r$-value, after $10^{7}$ transients are discarded.

Figure 3: Model 3 behaviour for $c=0.65 . p=10^{-3}$ throughout, and $x_{0}=0.1, y_{0}=0.0$, for each value of $r$.

Regarding the ecological implications of Model 3, we observe that just as was the case in Model 2, the introduction of a mutant predator can be stabilising, this time for sections of the range $1.5<c<6,3.6<r<4$. Moreover, it is dependent on the precise value of $r$ and the initial conditions, but we can see that this variant may permit stable coexistence in small areas of the parameter space where $r>4$ and thus $x$ would be driven to extinction altogether if it did not have the ability to create its own predator.

An example Feigenbaum diagram for this system, with $c=0.65$, and the plot of the corresponding Lyapunov exponents are given in Figure 3. Note that this Feigenbaum diagram (b) is fairly robust to variation in the system's initial conditions, being visually indistinguishable for $x_{0}=0.1,0.2, \ldots, 0.9$ with $y_{0}=0$. Observe the sustained region where the maximal exponent is zero, corresponding to the quasiperiodic behaviour. This is distinct from the non-differentiable point at $r \simeq 3.78$ where the exponent rises to zero, which instead (as can be seen from the Feigenbaum diagram) is due to a flip bifurcation from a Period-7 to a Period-14 orbit occuring within the next Arnol'd tongue with which this cross-section of the parameter space interacts. 


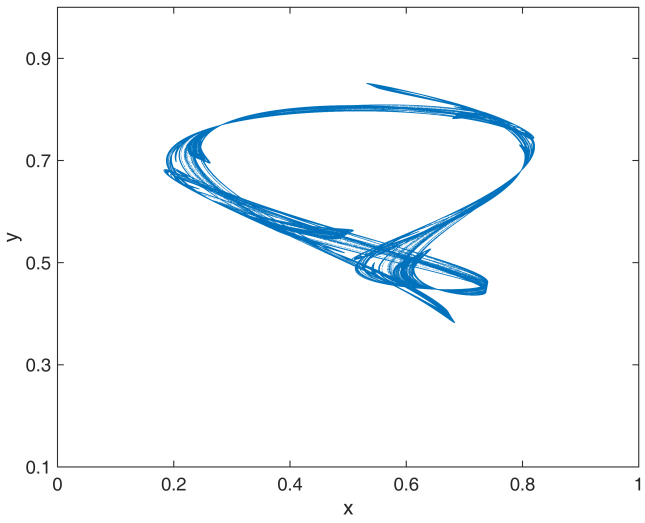

(a) $r=3.9300$

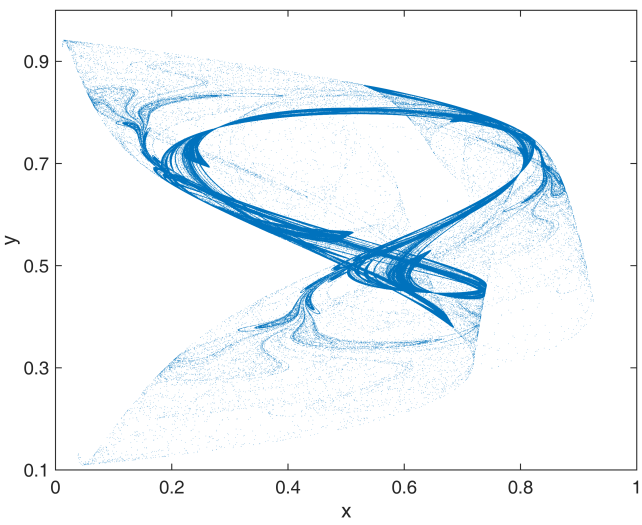

(c) $r=3.9350$

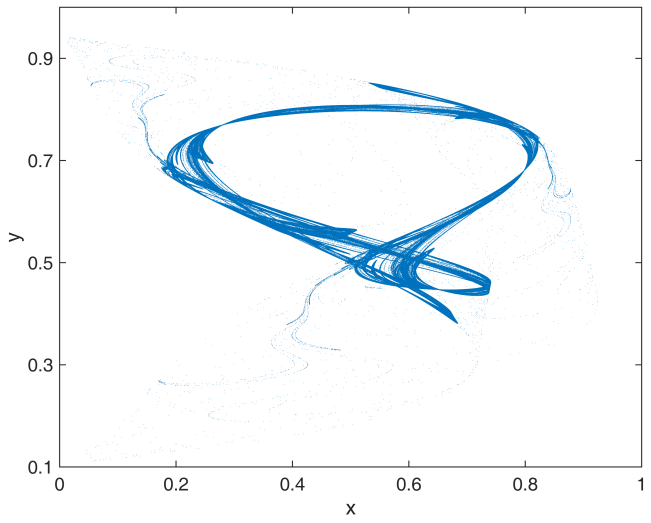

(b) $r=3.9325$

Figure 4: Model 3 - Strange Attractor, for $c=0.65$ and $p=10^{-3}$. In each case $5 \times 10^{6}$ iterations are plotted after $10^{7}$ transients are discarded.

Figure 3(c) provides a closer look at the diffuse wings that appear on the Feigenbaum diagram for $3.93<r<3.95$. The sparsity results from the frequency distribution of the orbits in phase space - for values of $r$ in this range the frequency (tested over $10^{9}$ iterations following $10^{7}$ transients) is low but non-zero at the extremes of the attractor. For example at $r=3.94$ the probability of visiting $0<x<0.1$ is 0.0025 . Consequently, very large orbits at each increment of $r$ would be required to produce definitive boundaries on the bifurcation diagram, well in excess of the 1000 iterations plotted in Figure 3(b). Moreover, an examination of the time-series within this range indicates that at approximately $r=3.9325$ the strange attractor undergoes a transition to one which fills a larger region of phase space. This process is illustrated in Figure 4.

Finally, numerical investigation indicates the presence of hyperchaos (that is, both Lyapunov exponents are strictly positive, which we take to be the case when both are greater than the error bound $10^{-3}$ ) for regions of the parameter space in Model 3 with $p=10^{-3}$. These regions are described in Figure 5, and Figure 6 shows an example of a hyperchaotic attractor that may be obtained from this system. 


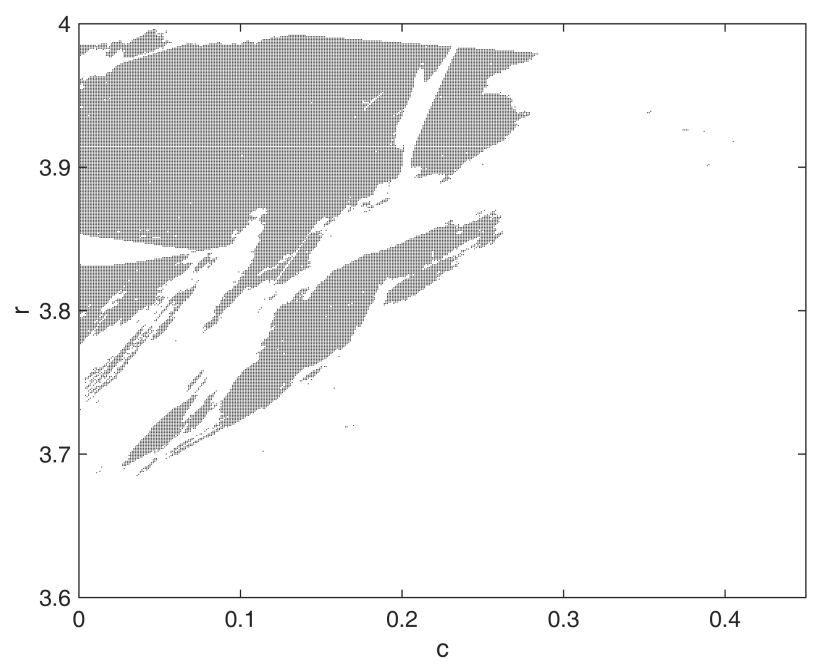

Figure 5: Model 3 Region of Hyperchaos. $p=10^{-3}$ throughout. For every set of parameter values $x_{0}=0.1, y_{0}=0.0$, and $10^{6}$ transients are discarded before both Lyapunov exponents are calculated over $10^{6}$ iterations.

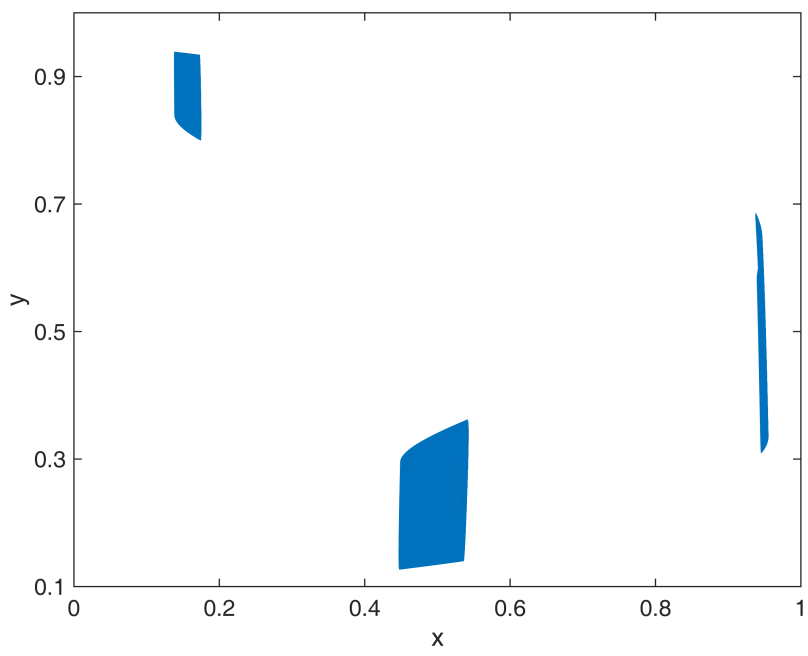

Figure 6: Model 3 Hyperchaotic Attractor, for $c=0.082, r=3.846 . p=10^{-3}, x_{0}=0.1$, and $y_{0}=0.0 .5 \times 10^{6}$ iterations are displayed after $10^{7}$ transients are discarded.

\section{The Limiting Case of Model 3 As $p \rightarrow 0$}

We now consider the limiting case of Model 3 with $p=0$ in greater detail. This has the benefit of far greater tractability for analytic results, and serves as an approximation for the system with a biologically realistic rate of mutation.

In this limit, the map is reduced to:

$$
\begin{aligned}
x_{n+1} & =r x_{n}\left(1-x_{n}\right)-c x_{n} y_{n} \\
y_{n+1} & =\frac{r}{2}\left(1+x_{n}\right) y_{n}\left(1-y_{n}\right)
\end{aligned}
$$

All numerical results presented in this section take the initial conditions $x_{0}=y_{0}=0.1$.

\subsection{Numerical Results}

The behaviour across the parameter space is illustrated in Figure 7, where the legend is altered to accomodate the new possibility present in this model - namely, the axial fixed point $\left(x^{*}, 0\right)$. The global structure is similar to that obtained for the case with $p$ non-zero, and so we are able to make some inferences about that more complex situation. The main changes in this case are: first, that the fractured upper boundary of the coexistence steady state is replaced by a smooth curve. 


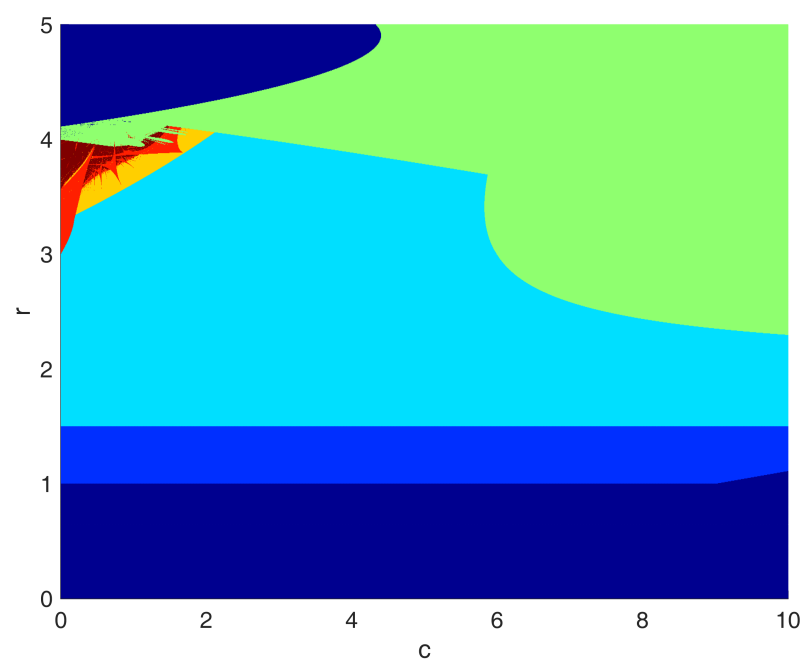

Figure 7: Model 3 limiting case with $p=0$ : Purple $=$ Extinction, Dark Blue $=x$-only Period 1, Light Blue $=$ Coexistence Period-1, Green = y-only Period 1, Orange $=2 \mathrm{D}$ Quasiperiodicity, Light Red $=2 \mathrm{D}$ Periodicity, Dark Red $=2 \mathrm{D}$ Chaos. For every set of parameter values $x_{0}=y_{0}=0.1$, and $10^{5}$ transients are discarded before the Lyapunov exponent is calculated over $10^{6}$ iterations.

Second, the range $1<r<3 / 2$ has been separated from the coexistence region. Here it is actually the axial fixed point $\left(1-\frac{1}{r}, 0\right)$ that is attracting, but in the case above where constant mutation was present there can of course never exist such an equilibrium. Observe that this is only fully the case for all of $1<r<3 / 2$ when $0<c<9$, as beyond this range the region is disrupted by the boundary $r=c / 9$, beneath which $x_{1}$ is negative. This results in full extinction for $0<c<18$ beneath this line, as the one-dimensional system governing $y$ alone does not have a non-trivial attractor for $r<2$. This boundary is dependent on initial conditions, such that if $x_{0}=y_{0}=\alpha$, then it is given by $r=\frac{c \alpha}{1-\alpha}$.

Finally, a curved region of total extinction has been introduced for approximately $0<c<4.1$ and $r>4.1$. This is found numerically to be the boundary of $y_{3}=1$, hence $y_{n}=0$ for $n \geq 4$ and so the recurrence relation for $x$ is reduced to the usual logistic equation. Since $r$ is greater than 4, $x$ then proceeds to go extinct following the standard properties of the logistic map. However, in this region in Figure 1(c) this feature was not present, which illustrates another benefit of even a low rate of mutation: it may allow the child species $y$ to persist to a steady state where we now see there would otherise be total extinction. This is because the region of extinction appearing in this case $(p=0)$ is caused by $y_{3} \geq 1$, leading $y$ to perish before $x$. Thus, $r>4$ causes $x$ to then also die out as stated. However, when mutation was present, $x$ was able to restart $y$ after the third time-step. $x$ subsequently dies out, leaving $y$ to attain the non-trivial fixed point of its one-dimensional dynamics which is stable throughout $2<r<6$.

\subsection{Analytic Results}

The system (13)-(14) has potentially four fixed points: $(0,0),\left(0,1-\frac{2}{r}\right),\left(1-\frac{1}{r}, 0\right)$, and $\left(x^{*}, y^{*}\right)$, where $\left(x^{*}, y^{*}\right)$ satisfies

$$
\begin{aligned}
r\left(1-x^{*}\right)-c y^{*} & =1 \\
r\left(1+x^{*}\right)\left(1-y^{*}\right) & =2 .
\end{aligned}
$$

The general Jacobian matrix is therefore given by:

$$
J(x, y)=\left(\begin{array}{cc}
r(1-2 x)-c y & -c x \\
\frac{r}{2} y(1-y) & \frac{r}{2}(1+x)(1-2 y)
\end{array}\right)
$$

In all that follows, we only consider solutions where $r>0$ and $c>0$ and the resulting $(x, y) \in[0,1) \times[0,1)$.

\subsection{Fixed Points and their Stability}

For the trivial fixed point $(0,0)$,

$$
J(0,0)=\left(\begin{array}{ll}
r & 0 \\
0 & \frac{r}{2}
\end{array}\right)
$$


and clearly the eigenvalues are $\Lambda_{1}=r$ and $\Lambda_{2}=\frac{r}{2}$, so it follows that

$$
(0,0) \text { is } \begin{cases}\text { stable } & \text { if } 0<r<1 \\ \text { a saddle point } & \text { if } 1<r<2 \\ \text { a repellor } & \text { if } 2<r\end{cases}
$$

Let us next analyse the axial fixed points.

The fixed point $\left(0,1-\frac{2}{r}\right)$ exists (and is distinct) precisely if $2<r$. Then,

$$
J\left(0,1-\frac{2}{r}\right)=\left(\begin{array}{cc}
r-c+\frac{2 c}{r} & 0 \\
1-\frac{2}{r} & \frac{r}{2}\left(\frac{4}{r}-1\right)
\end{array}\right)
$$

Thus the eigenvalues are $\Lambda_{1}=r-c+\frac{2 c}{r}$ and $\Lambda_{2}=\frac{r}{2}\left(\frac{4}{r}-1\right)=2-\frac{r}{2}$. Hence, $\left|\Lambda_{2}\right|<1$ for $2<r<6$, and for a given value of $c>0,\left|\Lambda_{1}\right|<1$ if $r>\frac{2 c}{c-2}$ for $0<c<2$ and if $r<\frac{2 c}{c-2}$ for $2<c$.

We therefore conclude that this fixed point is:

$$
\begin{cases}\text { stable } & \text { if } c<3, r<6, \text { or } 3<c, r<\frac{2 c}{c-2} \\ \text { a repellor } & \text { if } 2<c \text { and } r>\max \left(6, \frac{2 c}{c-2}\right) ; \\ \text { a saddle point } & \text { for } r>2 \text { otherwise. }\end{cases}
$$

The other axial fixed point $\left(1-\frac{1}{r}, 0\right)$ exists (and is distinct) precisely if $1<r$. Then,

$$
J\left(1-\frac{1}{r}, 0\right)=\left(\begin{array}{cc}
2-r & -c\left(1-\frac{1}{r}\right) \\
0 & r-\frac{1}{2}
\end{array}\right)
$$

Thus the eigenvalues are $\Lambda_{1}=2-r$ and $\Lambda_{2}=r-\frac{1}{2}$. Hence, $\left|\Lambda_{1}\right|<1$ precisely if $1<r<3$, and $\left|\Lambda_{2}\right|<1$ if $1<r<\frac{3}{2}$ for $1<r$.

We therefore deduce that this fixed point is:

$$
\begin{cases}\text { stable } & \text { if } 1<r<\frac{3}{2} \\ \text { a saddle point } & \text { if } \frac{3}{2}<r<3 \\ \text { a repellor } & \text { if } 3<r\end{cases}
$$

Finally, we consider the interior fixed point $\left(x^{*}, y^{*}\right)$.

First, we must determine when both co-ordinates lie on the real line. Simultaneously solving equations (15) and (16), and using $r>0$, we obtain the following solution for $x^{*}$ :

$$
x^{*}=\frac{1}{2 r}(-(c+1) \pm \sqrt{g(c, r)})
$$

where $g(c, r)$ is given by:

$$
g(c, r)=4 r^{2}+(-4-4 c) r+\left(c^{2}+10 c+1\right) .
$$

Solving $g(c, r)=0$ for a fixed value of $c$, we find that

$$
r=\frac{1}{2}(1+c \pm \sqrt{-8 c})
$$

Since we only consider $c>0$ there are no real, positive values of $r$ and $c$ such that $g(c, r)=0$. Now, $g$ is continuous and non-zero $\forall c, r>0$, so $g(1,1)=8>0$ implies that $g(c, r)>0$ and hence $x^{*}$ real for all $c, r>0$.

However, as we are investigating these as ecologically-inspired models, we restrict our interest to the range $0<x^{*}, y^{*}<1$. Clearly, for $x^{*}$ to be positive, we may only consider the positive root of (22). Now, substituting $x_{+}^{*}$ back into (15), we obtain

$$
y_{+}^{*}=\frac{1}{2 c}(2 r+c-1-\sqrt{g(c, r)}),
$$

and so $y_{+}^{*}$ is also real for all $c, r>0$. Next, we must establish for which parameters $\left(x_{+}^{*}, y_{+}^{*}\right)$ satisfy the ecological conditions. First,

$$
x_{+}^{*}=\frac{1}{2 r}(-(c+1)+\sqrt{g(c, r)})>0 \text { precisely if } \sqrt{g(c, r)}>c+1 .
$$

It follows that this condition is equivalent to

$$
c>\frac{r(1-r)}{2-r} \text { for } r<2, \text { and } c<\frac{r(1-r)}{2-r} \text { for } 2<r .
$$


(Note that the intersection of this curve with the boundary $r=c / 9$ reveals that the maximum predation rate which will permit coexistence in this system $(p=0)$ is $c=19.125$.)

Next, $x_{+}^{*}<1$ if and only if $\sqrt{g(c, r)}<2 r+c+1$, and hence precisely if:

$$
r>\frac{c}{1+c}
$$

For the $y$-co-ordinate, $y_{+}^{*}>0$ if $\sqrt{g(c, r)}<2 r+c-1$. This condition reduces to $r>\frac{3}{2}$. Finally, $y_{+}^{*}<1$ is satisfied if and only if $\sqrt{g(c, r)}>2 r-c-1$, and this requirement is met for all values of $r$ provided $c>0$.

From these conditions, we deduce that the interior fixed point $\left(x^{*}, y^{*}\right)$ is real, unique and lies in $(0,1) \times(0,1)$ in the following two regions of parameter space: $\frac{3}{2}<r<2$, and $2<r$ with $c<\frac{r(1-r)}{2-r}$. Given satisfactory values of $c$ and $r$, the fixed point is determined by:

$$
\begin{aligned}
x^{*} & =\frac{1}{2 r}(-(c+1)+\sqrt{g(c, r)}) \\
y^{*} & =\frac{1}{2 c}(2 r+c-1-\sqrt{g(c, r)})
\end{aligned}
$$

Next we shall investigate the linear stability of this fixed point, and the the associated bifurcations that may occur in this map.

\subsection{Bifurcation of the Interior Fixed Point}

We may study the stability of the interior fixed point using the Jury conditions of stability for a discrete system. Let $\Lambda \in \mathbb{C}$, and define

$$
F(\Lambda)=\Lambda^{2}-\operatorname{tr}\left(J\left(x^{*}, y^{*}\right)\right) \Lambda+\operatorname{det}\left(J\left(x^{*}, y^{*}\right)\right)
$$

where $t r$ and det denote the trace and determinant of the matrix respectively. Then $\left(x^{*}, y^{*}\right)$ is linearly stable precisely if these three conditions are satisfied [Ela05]:

$$
F(-1)>0, \quad F(1)>0, \quad 1-\operatorname{det}\left(J\left(x^{*}, y^{*}\right)\right)>0
$$

For our system, (13), (14), we find that the boundaries of these requirements are given by the following $6^{\text {th }}$-order polynomials:

$$
\begin{gathered}
0=8 r^{3}-8 r^{2}-4 c r^{2}+8 c r-2 r c^{2}+62 c+22 c^{2}+5+c^{3}+\sqrt{g(c, r)}\left(-7-2 c^{2}-15 c+2 c r+2 r-4 r^{2}\right) \\
0=8 r^{3}-8 r^{2}-4 c r^{2}+16 c r-2 r c^{2}+2 c+10 c^{2}+1+c^{3}+\sqrt{g(c, r)}\left(5-2 c^{2}-15 c+2 c r+2 r-4 r^{2}\right) \\
0=8 r^{3}-8 r^{2}-4 c r^{2}+12 c r-2 r c^{2}+16 c+16 c^{2}+3+c^{3}+\sqrt{g(c, r)}\left(-1-2 c^{2}-15 c+2 c r+2 r-4 r^{2}\right)
\end{gathered}
$$

Within the parameter space that concerns us (that is, where the fixed point exists), a numerical search returns the curves shown in Figure 8. The smaller, leftmost curve that exists only for $0<c<0.2$ gives the boundary of the condition $F(-1)>0$. Crossing this boundary corresponds with a real eigenvalue equal to -1 for the fixed point, resulting in a period-doubling bifuration and, as observed for the same region in Figure 7, the transition from a stable fixed point to periodic orbits.

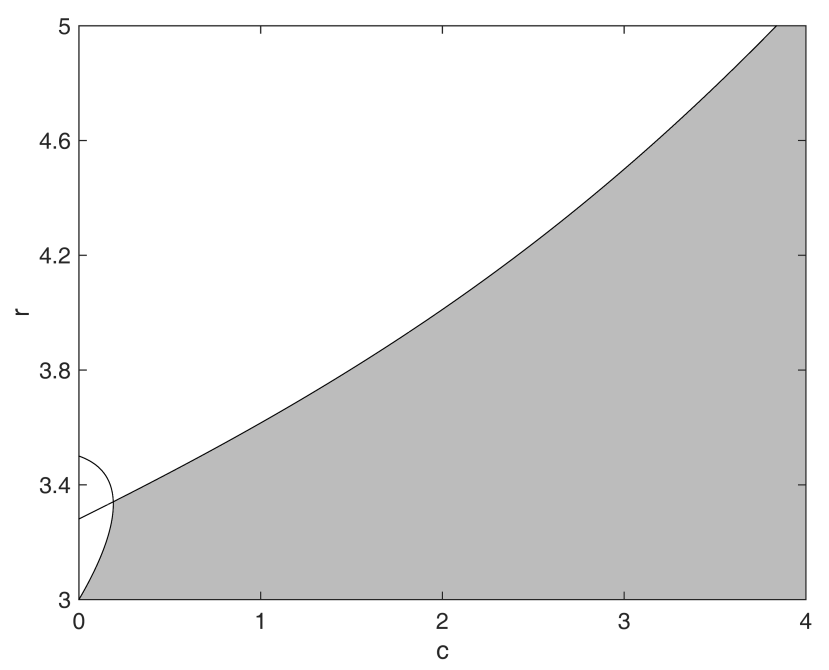

Figure 8: Jury Condition Boundary Curves 
The second curve that spans the range of $c$ shown is the boundary of $1-\operatorname{det}\left(J\left(x^{*}, y^{*}\right)\right)>0$. When this curve is crossed from a region of stability, but neither of the other two conditions are also satisfied, the eigenvalues have product and modulus 1 but they are not equal to \pm 1 , hence both eigenvalues are exiting the unit circle simultaneously and are complex conjugate. This indicates a Neimark-Sacker bifurcation, producing a closed invariant curve around the fixed point (see [Kuz13] for a suitable treatment of this topic, prevalent in many low-dimensional systems - for example a simple modification of the classic Hénon map [ES08b]). This accords with the numerical results which indicate the onset of quasiperiodic orbits in the region beyond this boundary, corresponding to the invariant curve possessing an irrational rotation number $\rho$. In such a case, any orbit is dense in the curve and fills it uniformly, an application of the equidistribution theorem that follows as a corollary of Weyl's criterion [KN12]. In the event of $\rho$ rational, the resulting periodic behaviour is maintained by the phase locking phenomenon as the parameters are varied further. These Arnol'd (resonance) Tongues of periodic behaviour are visible in the image, and are clearly seen in the zoomed image (Figure 2) in the case of mutation in the previous section. We infer that qualitatively similar behaviour is occuring in that case.

It appears that $F(1)>0$ is satisfied throughout the region. It follows that the interior fixed point is stable in the region that is bounded above by $r$ given as the minimum function of these two curves, shaded in Figure 8 .

Since this numerical analysis indicates that the equilibrium is destabilised only by a smooth continuation of this curve for larger values of $c$, and our analysis in the previous subsection showed that it continues to exist with biologically-reasonable values for all greater $r$ and $c$, we then turn our attention to the last unexplained feature of the parameter space: the smooth curve (replacing the shattered boundary for the case of $p=10^{-3}$ ) marking the collapse of the coexistence state to the axial fixed point $\left(0,1-\frac{2}{r}\right)$ for $2<c<6$. This is a separate occurrence from the parabola for approximately $6<c$, which is accounted for by equation (26) where the fixed point value $x^{*}$ is negative. From a further numerical search (Figure 9 shows values of $c, r$ satisfying $1-r\left(\frac{9 r-c}{100}\right)\left(1-\frac{9 r}{100}-\frac{79 c}{2000}\right)-\frac{99 c r}{4000}\left(1+\frac{9 r-c}{100}\right)\left(1-\frac{99 r}{2000}\right)=0$ within a tolerance of $\left.10^{-6}\right)$, we determine that this final boundary of the interior fixed point is the curve satisfying $x_{3}=0$ given these initial conditions $x_{0}=y_{0}=0.1$.

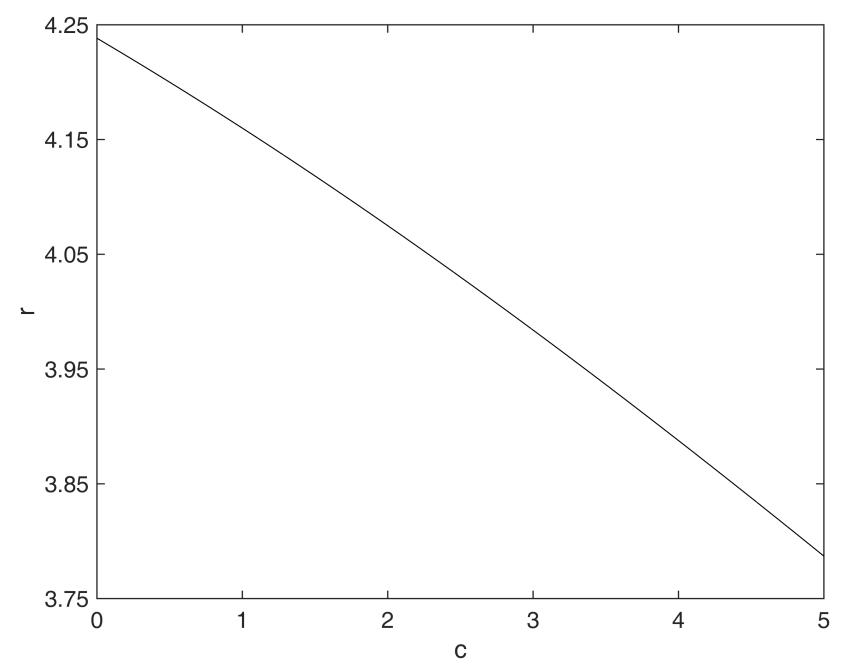

Figure 9: $x_{3}=0$ Boundary Curve

\section{Conclusion}

In this article we have studied a selection of simple discrete two-dimensional population models, that could be considered as toy models encapsulating the effects of predation and mutation in the simplest reasonable manner. A variety of interesting phenomena may be observed, including chaotic dynamics, hyperchaotic attractors, and Neimark-Sacker bifurcation producing quasiperiodic orbits and Arnol'd tongues with a fractal-like structure in parameter space. These models also illustrate several forms of beneficial self-limitation, such that the production of a mutant predator can allow the prey species to survive, or to attain a stable population, where it otherwise could not. Although a highly abstracted coupled logistic map, the model demonstrates that such ecological phenomena can, in principle, occur in nature without necessitating external perturbations or greater sophistication.

For greater justification as an ecological model, several improvements are suggested. More realistic Ricker growth could be used for the prey, and the growth function (numerical response) of the predator species should be dependent on the functional response (which could be replaced with Holling Type II) and, in particular, on the predation parameter $c$. This 
would be a clear next step for such an investigation. However, one risks introducing too much complexity and indeed even for the present models the scope of analytic statements is fairly restricted - such that one must consider the limiting case of $p \rightarrow 0$ in order to obtain any useful results. For the researcher interested in pursuing these themes on an ecologically realistic scale, we recommend the work that has been underway in recent years to develop eco-evolutionary models of food webs such as [LL05, CHM98, DHM01]. These are computational models with stochastic elements that simultaneously model the effects of random mutation coupled with natural selection in the form of trait-dependent trophic interactions, in order to produce realistic and complex model food webs as emergent results of simulations.

\section{Acknowledgements}

G.M.A. is funded by the Department for Employment and Learning.

\section{References}

[AEEME09] H.N. Agiza, E.M. ELabbasy, H. EL-Metwally, and A.A. Elsadany. Chaotic dynamics of a discrete prey-predator model with Holling type II. Nonlinear Anal.-Real., 10(1):116 - 129, 2009.

[AG89] Roger Arditi and Lev R. Ginzburg. Coupling in predator-prey dynamics: Ratio-dependence. J. Theor. Biol., 139(3):311 - 326, 1989.

[AG00] Peter A. Abrams and Lev R. Ginzburg. The nature of predation: prey dependent, ratio dependent or neither? Trends Ecol. Evol., 15(8):337 - 341, 2000.

[AM16] Gavin M Abernethy and Mark McCartney. Cannibalism and chaos in the classroom. Int. J. Math. Educ. Sci. Technol., page In press, 2016.

[BCPS01] Denis Blackmore, Jerry Chen, John Perez, and Michelle Savescu. Dynamical properties of discrete Lotka-Volterra equations. Chaos Solitons Fractals, 12(13):2553 - 2568, 2001.

[Bed75] JR Beddington. Mutual interference between parasites or predators and its effect on searching efficiency. J. Anim. Ecol., 44(1):331-340, 1975 .

[BLR10] B. Buonomo, D. Lacitignola, and S. Rionero. Effect of prey growth and predator cannibalism rate on the stability of a structured population model. Nonlinear Anal.-Real., 11(2):1170 - 1181, 2010.

[CHM98] Guido Caldarelli, Paul G. Higgs, and Alan J. McKane. Modelling coevolution in multispecies communities. J. Theor. Biol., 193(2):345 - 358, 1998.

[DHM01] Barbara Drossel, Paul G. Higgs, and Alan J. McKane. The influence of predator-prey population dynamics on the long-term evolution of food web structure. J. Theor. Biol., 208(1):91 - 107, 2001.

[Ela05] Saber Elaydi. An Introduction to Difference Equations. Springer Science \& Business Media, 2005.

[ES08a] Zeraoulia Elhadj and J C Sprott. The effect of modulating a parameter in the logistic map. Chaos, 18(2):023119, Jun 2008.

[ES08b] Zeraoulia Elhadj and Julien Clinton Sprott. A minimal 2-d quadratic map with quasi-periodic route to chaos. Int. J. Bifurcat. Chaos, 18(05):1567-1577, 2008.

[Fro92] Jan Froyland. Introduction to chaos and coherence. CRC Press, 1992.

[Hen97] Shandelle M. Henson. Cannibalism can be beneficial even when its mean yield is less than one. Theor. Pop. Biol., 51(2):109 - 117, 1997.

[HIT10] A N W Hone, M V Irle, and G W Thurura. On the Neimark-Sacker bifurcation in a discrete predator-prey system. J. Biol. Dyn., 4(6):594-606, Nov 2010.

[HL11] Zhimin He and Xin Lai. Bifurcation and chaotic behavior of a discrete-time predator-prey system. Nonlinear Anal.-Real., 12(1):403 $-417,2011$.

[Hol59] C. S. Holling. Some characteristics of simple types of predation and parasitism. Can. Entomol., 91:385-398, 7 1959.

[HTZ11] Zengyun Hu, Zhidong Teng, and Long Zhang. Stability and bifurcation analysis of a discrete predator-prey model with nonmonotonic functional response. Nonlinear Anal.-Real., 12(4):2356 - 2377, 2011.

[JY06] Zhujun Jing and Jianping Yang. Bifurcation and chaos in discrete-time predator-prey system. Chaos Solitons Fractals, 27(1):259 277, 2006.

[KE95] C. Kohlmeier and W. Ebenhöh. The stabilizing role of cannibalism in a predator-prey system. B. Math. Biol., 57(3):401-411, 1995.

[KG14] R. Khoshsiar Ghaziani. Dynamics and bifurcations of a Lotka-Volterra population model. Iran. J. Sci. Technol. (Sci.), 38(3):265-279, 2014 .

[KN12] Lauwerens Kuipers and Harald Niederreiter. Uniform Distribution of Sequences. Courier Corporation, 2012.

[KT03] C. Kaewmanee and I.M. Tang. Cannibalism in an age-structured predator-prey system. Ecol. Model., $167(3): 213-220,2003$.

[Kuz13] Yuri A Kuznetsov. Elements of Applied Bifurcation Theory, volume 112. Springer Science \& Business Media, 2013.

[LL05] Nicolas Loeuille and Michel Loreau. Evolutionary emergence of size-structured food webs. Proc. Natl. Acad. Sci., 102(16):5761-5766, 2005 .

[Llo95] Alun L. Lloyd. The coupled logistic map: a simple model for the effects of spatial heterogeneity on population dynamics. Journal of Theoretical Biology, 173(3):217 - 230, 1995.

[SJL10] Yasuhisa Saito, Changdo Jung, and Yongdo Lim. Effects of cannibalism on a basic stage structure. Appl. Math. Comput., 217(5):2133 $-2141,2010$.

[Smi68] J. Maynard Smith. Mathematical Ideas in Biology. Cambridge University Press, 1968.

[Spr03] Julien Clinton Sprott. Chaos and Time-Series Analysis, volume 69. Oxford University Press Oxford, 2003.

[Vol26] Vito Volterra. Fluctuations in the abundance of a species considered mathematically. Nature, 118:558-560, 1926. 